\title{
On the Applicability of Image Processing Techniques in the Radio Environment Characterisation
}

\author{
J. Pérez-Romero, O. Sallent, R. Agustí \\ Dept. Signal Theory and Communications (TSC) \\ Universitat Politècnica de Catalunya (UPC), Barcelona, Spain \\ E-mail: [jorperez,sallent, ramon]@tsc.upc.edu
}

\begin{abstract}
This paper presents a novel framework based on image processing techniques for the radio environment characterisation. Specifically, after digitalising a given scenario in accordance with the frequencies that are detected in each point, an image can be built whose pixel intensities capture the radio-electrical conditions. Through non-linear filtering and object detection operations the proposed framework allows identifying the homogeneous regions where certain frequencies can be detected. This methodology can be used in different contexts, such as the construction of databases to provide the terminals with spectrum awareness in flexible spectrum scenarios to facilitate the switch-on procedure or the secondary usage of spectrum, the support to devise proper strategies for cell and RAT selection, etc.
\end{abstract}

Keywords-image processing; radio access technologies; radio environment characterisation

\section{INTRODUCTION}

Recent years have witnessed the evolution of a large plethora of wireless technologies with different characteristics, as a response of the operators' and users' needs in terms of an efficient and ubiquitous delivery of advanced multimedia services. As a result, current and future wireless scenarios will be characterised by a multiplicity of Radio Access Technologies (RATs) and network operators with very different deployments (e.g. cellular, wireless local area networks, etc.). In addition to this, and with the objective of ensuring an efficient utilisation of the available spectrum bands, the regulatory perspective on how the spectrum should be allocated and utilized is evolving as well [1]. In this sense, the traditional fixed spectrum assignment to a licensee which has exclusive exploitation rights for a particular spectrum range may not be satisfactory to respond to the new radio use context that requires enhancement in spectrum efficiency and can lead to spectrum underutilization [2]. Then, instead of the classical fixed spectrum assignment to licensed systems and services, which may become too rigid and inefficient, the possibility to use flexible spectrum management strategies that dynamically allocate spectrum bands in accordance with the specific traffic needs and RAT deployments in each area is being recently considered [3][4]. Similarly, new technical advances are focused on the development of strategies and policies aiming to the utmost and efficient access to shared spectrum resources. As an example, the unlicensed use of VHF and UHF TV bands by secondary users, provided no harmful interference is caused to the licensee (i.e. primary user), was targeted by the FCC in [5].

As a result of the above trends, wireless scenarios with a multiplicity of RATs and operators, and exploiting flexible spectrum management strategies, will exhibit a high degree of complexity affecting not only the network operation (e.g. which are the possible interferers at each point) but also the capability of mobile terminals to identify the available networks. This capability can be referred to as spectrum awareness, which corresponds to the mechanisms allowing the terminal to be aware of the communication means available at a given time and place.

In this flexible spectrum management framework, where a number of transceivers with flexible time-varying assignment of operating frequency and/or RAT are deployed, spectrum awareness from the mobile perspective arises as a challenge. Different techniques have been proposed in the literature to provide the necessary spectrum awareness to mobiles. Some of them rely on sensing mechanisms in which the mobile devices try to identify, either by themselves or through a proper cooperation with other devices, which are the available RATs and frequencies in a given area [6][7]. However, these mechanisms suffer from long measurement times if the bandwidth to be sensed is very large, inaccuracies in the measurements due to different effects (e.g. channel fading, hidden terminal, etc.), high complexity and power consumption at the mobile, etc. On the other extreme, other proposed mechanisms are based on signalling channels through which the network can provide different types of information regarding the spectrum and RAT occupancy in different areas. Some examples falling in this category of solutions are the Common Spectrum Coordination Channel (CSCC) [8], the Spectrum Information Channel (SIC) [9] and the Cognitive Pilot Channel (CPC) concept [10]. One of the key aspects for the success of these solutions is having appropriate up-to-date databases containing the relevant aspects of radio environment characterisation. An example of these databases is the so-called Radio Environment Map (REM) in [11].

The characterisation of the radio environment in a given scenario is in general a complex task due to the multiple effects affecting radio propagation and the different natures of the considered access technologies involving very different ranges, power levels, etc. In that sense, one possibility to capture all this information in the form of a database consists in identifying geographical regions in which certain radioelectrical homogeneities can be defined (e.g. the mesh concept considered in [10]), so that the information stored in the database (e.g. the set of frequencies and RATs that are being detected) is valid for all the points within a given region. Such a characterisation of the environment can simplify the signalling of the awareness information through channels such 
as the CPC and, on the other hand, can be useful to decide appropriate deployments of the awareness networking mechanisms (e.g. the ranges, bandwidths and power levels of the CPC transmitters, etc.). The construction and maintenance of the mentioned database can be envisaged in many different forms (e.g. with the support of planning tools, measurement reports from mobile terminals, sensor networks, etc.).

Under the above considerations, this paper presents an innovative framework to cope with the characterisation of homogeneous radio-electrical regions in a given scenario. The problem is tackled from a multi-disciplinary perspective by transforming the radio-electrical conditions of the scenario into a digitalised image and then applying well-known techniques in the field of image processing, such as object detection and non-linear filtering in order to characterise the radio-electrical homogeneous regions. It is worth noting that the proposed methodology intends to provide a framework for a quantitative analysis of the resulting areas with homogeneous conditions in the radio environment (i.e. number of such areas, corresponding area size, etc.) and, therefore, the resulting characterisation can be of interest for a number of engineering techniques (e.g. to build up a database to support flexible spectrum scenarios, to devise proper strategies for cell and RAT selection and reselection strategies, support to camping strategies, etc.).

The rest of the paper is organized as follows. Section II presents in more detail the problem, while Section III proposes the new image processing-based methodology for radio environment characterisation. Section IV presents some illustrative results in different scenarios while Section V summarises the conclusions.

\section{PROBlem Definition AND OBJeCtIVES OF THE PAPER}

Let assume a generic scenario such as the one depicted in Figure 1. It is characterised by a number of transmitters corresponding to different RATs which operate at different frequencies and having very different coverage areas (e.g. the central transmitter operating in a broadcast-like RAT with an extensive coverage area at frequency f5, or the transmitters operating at RATs 1 and 2 with frequencies f1, f2, f3 and f4 that could correspond to some cellular-like RATs). Clearly, by considering the overlapping in the coverage areas of all the transmitters different regions can be identified.

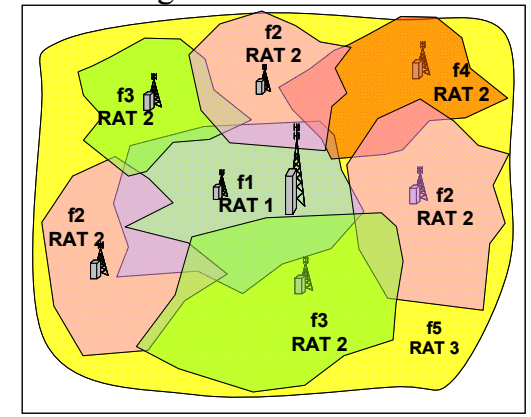

Figure 1 Generic scenario with different RATs and frequencies.

Then, assuming that the corresponding power levels of each transmitter and RAT in each geographical point are known (e.g. they could have been obtained by either a planning tool, through measurements, etc.), the objectives of this paper can be summarised as:

1.- To propose a systematic methodology to identify and characterise the homogeneous regions in a multi-RAT and multi-frequency scenario

2.- To obtain the typical sizes of the homogeneous regions in different deployments.

\section{IMAGE PROCESSING -BASED METHODOLOGY}

The proposed methodology starts from the scenario deployment characterised in terms of the power levels that can be measured at each specific frequency in each geographical point. In that respect, let define $P\left(f_{i}, x, y\right)$ as the power that is measured from frequency $f_{i}$ in the geographical coordinates $(x, y)$. Then, the following steps are carried out.

\section{A. Radio environment digitalisation into an image}

The first step consists in digitalising the power levels building an image. For that purpose, assuming that from a spectrum awareness point of view it is only relevant to know if in a specific position a frequency is being detected or not, rather than the specific value of the power received, let define the binary representation of frequency $f_{i}$ in coordinates $(x, y)$ as:

$$
M\left(f_{i}, x, y\right)=\left\{\begin{aligned}
1 & \text { if } f_{i} \text { detected in }(x, y) \Leftrightarrow \text { if } P\left(f_{i}, x, y\right) \geq P_{t h}\left(f_{i}\right) \\
0 & \text { if } f_{i} \text { not detected in }(x, y) \Leftrightarrow \text { if } P\left(f_{i}, x, y\right)<P_{t h}\left(f_{i}\right)
\end{aligned}\right.
$$

where $P_{t h}\left(f_{i}\right)$ would be the detection threshold associated to the RAT transmitted at frequency $f_{i}$.

From the above representation, it is possible to characterise each geographical coordinate $(x, y)$ by a value corresponding to the sum of the binary representations of all the considered frequencies in the scenario:

$$
I(x, y)=\sum_{i=1}^{N} M\left(f_{i}, x, y\right) 2^{i-1}
$$

where $N$ is the total number of frequencies in the scenario. As an example, if we assume $N=3$ frequencies $f_{1}, f_{2}, f_{3}$, a point where the three frequencies are detected would be encoded as $I=7$, whereas a point in which only $f_{1}$ and $f_{3}$ are detected would be encoded as $I=5$.

Finally, by spatially sampling the $I(x, y)$ with horizontal and vertical sampling resolutions $\Delta x$ and $\Delta y$, respectively, a digital image in which the value of each pixel, i.e. a rectangular block of $\Delta x \times \Delta y$, would correspond to a colour-level associated with the value $I(x, y)$ depending on the frequencies that are detected inside this pixel. In this way, the radio-electrical homogeneous regions could be easily identified in the image as those sets of pixels having the same colour (i.e. the objects in the image).

\section{B. Non-linear image filtering}

Due to the irregularities caused by the radio propagation in real environments (e.g. shadowing effects), it may turn that some of the obtained images in the previous step exhibit a certain random behaviour in which some pixels can slightly differ from their neighbours because of being in the border coverage areas of certain RATs in which some coverage fluctuations may arise. This effect would be graphically observed as a blurred image, and would introduce some randomness in the contour of the identified objects. In order to 
reduce this effect, it is possible to make use of image filtering strategies that are able to "clean" the obtained image while keeping the more relevant propagation characteristics.

An image filtering technique consists in modifying the value of each pixel as a function of the values of the pixels in a certain neighbourhood around the considered pixel. Depending on how the function is defined, image filtering techniques can be either linear or non-linear. Among the non-linear techniques, morphological procedures such as the dilation or the erosion, in which each pixel gets the value of the maximum or the minimum pixel, respectively, in a given neighbourhood, are the most common ones [12].

In the context of the problem considered here, since we aim to preserve the radio-electrical characteristics captured in the binary-level representation $M\left(f_{i}, x, y\right)$, a non-linear filtering function has been considered to derive the filtered value of each pixel $I_{f}(x, y)$ based on the prior filtering of the components $M\left(f_{i}, x, y\right)$ in accordance with the neighbourhood. Specifically, the filtered binary-level representations are:

$M_{f}\left(f_{i}, x, y\right)= \begin{cases}1 & \text { if } f_{i} \text { detected in a fraction } \geq \theta \text { of pixels in } N(x, y) \\ 0 & \text { if } f_{i} \text { detected in a fraction }<\theta \text { of pixels in } N(x, y)\end{cases}$

where $N(x, y)$ is the neighbourhood of pixel $(x, y)$, defined as a square area having the pixel $(x, y)$ in the centre, with dimensions $N_{\text {size }} \times N_{\text {size }}$ pixels. In turn, $\theta$ is a parameter of the filter whose value will be set depending on the desired reliability of the frequency availability information in the scenario. The impact of this parameter will be further discussed in the results in section IV.B.

Finally, the pixels in the filtered image are given by:

$$
I_{f}(x, y)=\sum_{i=1}^{N} M_{f}\left(f_{i}, x, y\right) 2^{i-1}
$$

\section{Object detection}

The third step in the proposed methodology consists in identifying those regions in the filtered image characterised by the same pixel values. These are the so-called objects and reflect the homogeneous areas with the same detected frequencies. This procedure is carried out following the socalled connected-component labelling technique [13] that consists in scanning the image and making groups of pixels (i.e. objects) based on pixel connectivity. Specifically, 4connected pixels are assumed, meaning that pixels are connected if their edges touch. Then, a pair of adjoining pixels is part of the same object only if they are both connected along the horizontal or vertical direction and they have the same value $I_{f}(x, y)$.

\section{Region measurement}

Once the different objects or homogeneous regions have been identified, the last step consists in obtaining the metrics of interest for each region. Specifically, in this work we are interested in the total area of each region, which can be computed based on the number of pixels existing in the region multiplied by the pixel area. However, other metrics such as the centroid or the diameter of a circle with the same area of the region could also be computed. From the areas of the identified regions the statistical distribution of the region size in the form of a probability density function (pdf) or Cumulative Distribution Function (CDF) can be retained as a parameter of interest to identify the dispersion in the region sizes and the typical region areas in each scenario. It is worth mentioning that, in order to avoid residual effects due to the randomness that have not been completely removed in the filtering phase, only regions having a minimum number of pixels $N_{\min }$ will be considered in the statistical computations.

\section{RESULTS}

This section presents some results in specific scenarios to illustrate the potentials of the proposed framework.

\section{A. Cellular scenario without shadowing effects}

Let assume first a cellular deployment with cell radius $1 \mathrm{~km}$ and hexagonal layout, with some overlapping between cells, and with a 3 frequency reuse pattern $\left(f_{1}, f_{2}, f_{3}\right)$. The total scenario size is $10 \mathrm{~km} x 10 \mathrm{~km}$, and the pixel size is $\Delta x=\Delta y=10 \mathrm{~m}$. Figure 2 plots the image corresponding to the digitalisation. Since only $N=3$ frequencies are considered, pixels are encoded according to (2) with $8=2^{N}$ different intensity levels (i.e. colours) where the value $7=111$ corresponds to the areas where three cells are overlapped, the values $3=011,5=101$ and $6=110$ corresponds to the areas where two cells overlap and finally the values $1=001,2=010$ and 4=100 correspond to the central areas of each cell.

Since this scenario corresponds to an ideal case in which propagation is not affected by shadowing effects, the different regions in the figure are very clearly identified and can be measured without the need of the non-linear filtering step, so that as a result of the process the CDF of the region size can be easily obtained as depicted in Figure 3. In this figure, three main region sizes can be identified, corresponding to the three sharp steps in the CDF. The first one, with an area of around $1.5 \mathrm{~km}^{2}$, corresponds to the regions where only 1 frequency is detected (i.e. the inner part of the different cells). The second one, with an area of around $0.3 \mathrm{~km}^{2}$, corresponds to the regions where two frequencies are detected (i.e. the areas where two cells overlap). Finally, the third main size is around $0.05 \mathrm{~km}^{2}$ and corresponds to the area where 3 cells overlap.

\section{B. Cellular scenario with shadowing effects}

When considering the same cellular scenario as in previous sub-section, but now with an additional shadowing in the propagation loss, the visual effect over the digitalised image can be observed in Figure 4, where it can be noticed that, although similar regions are identified, contours are not clearly defined and the image is blurred. To overcome this aspect and better analyse the image in terms of regions, it turns out to be convenient to apply the non-linear filtering explained in subsection III.B. The corresponding results, when a square neighbourhood with $N_{\text {size }}=5$ is considered, are plotted in Figure 5 for two different values of the filter parameter $\theta$, namely $\theta=0.95$ and $\theta=0.15$. Similarly, the resulting CDFs of the detected region sizes in the two cases are plotted in Figure 6, where only the regions having an area above $N_{\min }=100$ pixels have been considered. 


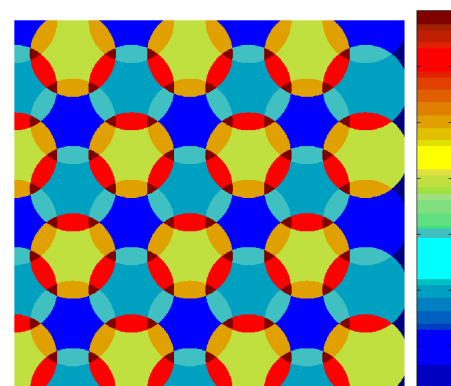

Figure 2 Image of the ideal cellular scenario without shadowing. In the right part, the colour scale of each pixel intensity between 0 and 7 is plot.

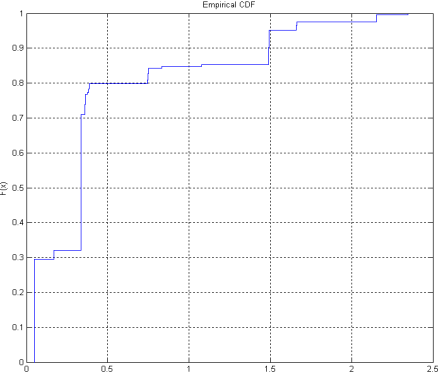

Figure $3 \mathrm{CDF}$ of the region size measured in $\mathrm{km}^{2}$.

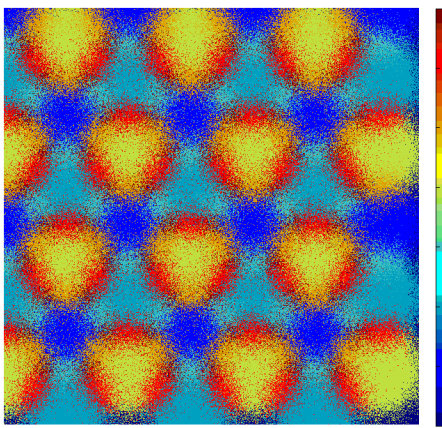

Figure 4 Image of the cellular scenario with $3 \mathrm{~dB}$ standard deviation shadowing. In the right part, the colour scale of each pixel intensity between 0 and 7 is plot.

Figure 5 shows that parameter $\theta$ plays an important role in the physical interpretation of the identified regions. Large values of $\theta$, such as in Figure 5(a), will tend to highlight areas where the same frequency is detected in all the neighbourhood. This leads to areas with few frequencies (i.e. most of the pixels correspond to the colours $1=001,2=010$ and $4=100$ where only a single frequency is identified) mainly located in the inner part of cells. Consequently, this can be relevant in case the regions to be identified are those where certain frequencies are detected with a very high reliability. For example, this could be useful in case that a channel such as CPC is to be used as a support for the switch-on procedure, because whenever a frequency is signalled for a given position, it is very likely that the mobile will actually measure it. Correspondingly, notice in Figure 6(a) that the identified region sizes tend to be large areas, in the order of the cell area.

On the other hand, low values of $\theta$, such as in Figure 5(b), will tend to highlight areas where a frequency is detected at some point of the neighbourhood, even if only in a few number of points. This leads to areas where multiple frequencies can be detected, mainly in the outer part of cells, and it can be graphically observed in the figure by the brown coloured pixels corresponding to the value $7=111$, where all three frequencies are detected in the areas where three cells overlap. Correspondingly, notice in Figure 6(b) that most of the identified region sizes tend to be small areas, in the order of magnitude of the overlapping area between two or three cells. Setting $\theta$ at low values could therefore be useful in case that this methodology is used to identify those areas where frequencies are forbidden for a secondary use. Notice that the signalling, e.g. through CPC, of the brown areas in Figure 6(b) would prevent secondary users from using any of the three frequencies in the scenario even if at some point one of this frequencies is not actually measured, because they could be generating interference to other neighbouring points where this frequency is actually detected.



(a)



(b)
Figure 5 Filtered image with (a) $\theta=0.95$, (b) $\theta=0.15$. In the right part, the colour scale of each pixel intensity between 0 and 7 is plot.

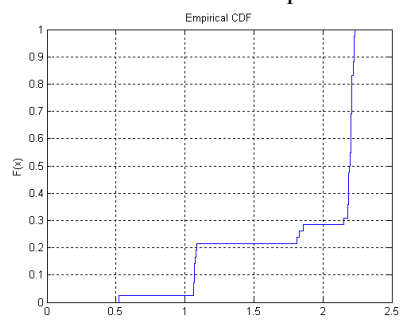

(a)

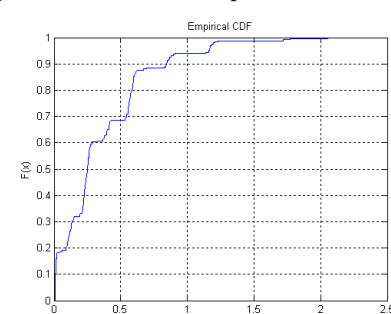

(b)
Figure $6 \mathrm{CDF}$ of the region size measured in $\mathrm{km}^{2}$ when (a) $\theta=0.95$, (b) $\theta=0.15$.

\section{Multi-RAT scenario}

Finally, to illustrate the capabilities of the proposed methodology in a more complex scenario, let assume that in the same cellular structure with three frequencies $\left(f_{1}, f_{2}, f_{3}\right)$ from the previous section, a wide range broadcast transmitter is added in the centre, with a coverage area of $4 \mathrm{~km}$, and operating with frequency $f_{4}$. In addition, a total of 13 WLAN hotspots with radius $200 \mathrm{~m}$ and operating at frequency $f_{5}$ are scattered in the scenario. Shadowing effects are also included in the propagation. The resulting digitalised image is shown in Figure 7 , where it should be observed that the colour scale has changed with respect to previous sub-sections because now the maximum pixel intensity corresponds to the points where all the five frequencies are detected, i.e. a value $31=11111$, that can be observed in some parts of the WLAN hotspots located inside the broadcast coverage area.

The resulting filtered image, for two different values of $\theta$, is shown in Figure 8, and the corresponding CDFs of the identified region sizes are plotted in Figure 9. Similar effects as those presented in sub-section IV.B can be observed. In 
particular, high values of $\theta$ tend to identify large areas where certain frequencies are detected with high reliability, corresponding to the inner parts of the different transmitters' coverage areas, while low values of $\theta$ tend to identify smaller areas where multiple frequencies are detected, mainly located in the edge points of the transmitters' coverage areas. It is worth mentioning that in both cases, the number of detected regions significantly increases with respect to the cellular case considered in previous sub-sections, reflecting the higher complexity of the multi-RAT scenario.

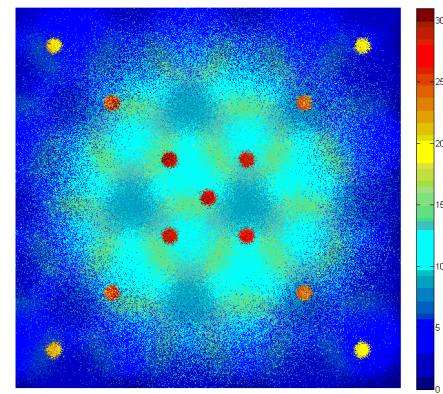

Figure 7 Image of the scenario with cellular, broadcast and WLAN technologies and shadowing effects. In the right part, the colour scale of each pixel intensity between 0 and 31 is plot.

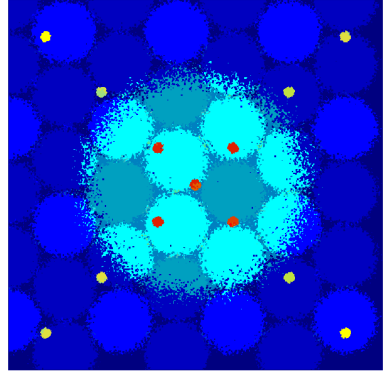

(a)

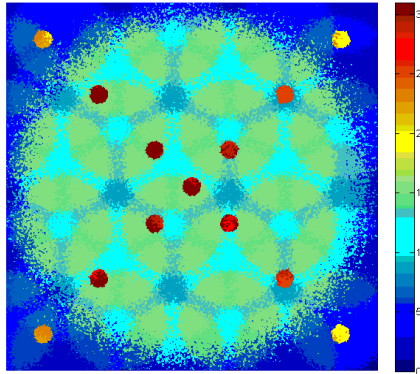

(b)
Figure 8 Filtered multi-RAT image with (a) $\theta=0.95$, (b) $\theta=0.15$. In the right part, the colour scale of each pixel intensity between 0 and 31 is plot.

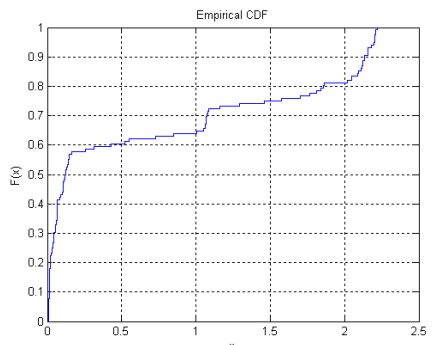

(a)

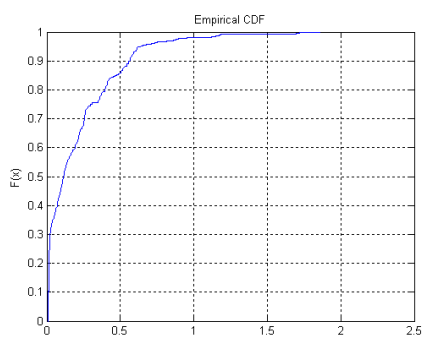

(b)
Figure $9 \mathrm{CDF}$ of the region size measured in $\mathrm{km}^{2}$ for the multi-RAT scenario when (a) $\theta=0.95$, (b) $\theta=0.15$.

\section{CONCLUSIONS}

This paper has presented a novel framework based on image processing techniques for the characterisation of homogeneous radio-electrical regions where certain frequencies can be detected. After digitalising the scenario in accordance with the frequencies that are detected in each point, an image is built whose pixel intensities capture the radioelectrical conditions. Then, through non-linear filtering and object detection operations, the proposed methodology is able to easily identify the homogeneous areas. A possible applicability of the proposed approach is the support to build databases containing frequency usage information that can be eventually signalled to mobile terminals to facilitate the switch on process in flexible spectrum management scenarios or to indicate frequencies that can be used by a secondary market without causing interference to primary receivers.

\section{ACKNOWLEDGEMENTS}

This work was performed in project $\mathrm{E}^{3}$ which has received research funding from the Community's Seventh Framework program. This paper reflects only the authors' views and the Community is not liable for any use that may be made of the information contained therein. The contributions of colleagues from $\mathrm{E}^{3}$ consortium are hereby acknowledged. This work has also been supported by the Spanish Research Council and FEDER funds under COGNOS grant (ref. TEC2007-60985).

\section{REFERENCES}

[1] M. Cave et al., Essentials of modern spectrum management, Cambridge University Press, 2007.

[2] I.F. Akyildiz, W.-Y. Lee, M.C. Vuran, S. Mohanty, "Next generation/dynamic spectrum access/cognitive radio wireless networks: a survey", Comput. Networks (Elsevier) 50 (13) (2006) 2127-2159.

[3] Qing Zhao; Sadler, B.M., "A Survey of Dynamic Spectrum Access," IEEE Signal Processing Magazine, vol.24, no.3, pp.79-89, May 2007.

[4] H. R. Karimi, T.W.Ho, H. Claussen, L. G. Samuel, "Evolution towards dynamic spectrum sharing in mobile communications", IEEE PIMRC Conference, Helsinki, September, 2006.

[5] Federal Communications Commission (FCC), "Notice of Proposed Rule Making," ET Docket no. 04-113, May 25, 2004.

[6] G. Ganesan, Ye Li; B. Bing, S. Li, "Spatiotemporal Sensing in Cognitive Radio Networks," IEEE JSAC, vol.26, no.1, pp.5-12, Jan. 2008

[7] A. Ghasemi, E.S. Sousa, "Collaborative spectrum sensing for opportunistic access in fading environments," DySPAN'05, 8-11 Nov.

[8] D. Raychaudhuri, X. Jing "A Spectrum Etiquette Protocol for Efficient Coordination of Radio Devices in Unlicensed Bands", 14th IEEE International Symposium on Persona1,lndoor and Mobile Radio Communication Proceedings (PIMRC), Beijing, September, 2003.

[9] M. Buddhikot, P. Kolodzy, S. Miller, K. Ryan, J. Evans “ DIMSUMNet: New Directions in Wireless Networking Using Coordinated Dynamic Spectrum Access", IEEE International Symposium on a World of Wireless, Mobile and Multimedia Networks (IEEE WoWMoM), Taormina/Giardini Naxos, Italy, June, 2005.

[10] J. Pérez-Romero, O. Sallent, R. Agustí, L. Giupponi, "A Novel OnDemand Cognitive Pilot Channel enabling Dynamic Spectrum Allocation', DySPAN '07, 17-20 Apr.

[11] Y. Zhao, D. Raymond, C. R. C. M. da Silva, J. H. Reed, and S. F. Midkiff, "Performance evaluation of radio environment map-enabled cognitive spectrum-sharing networks," in Proc. IEEE Military Comm. Conf., Orlando, FL, 2007, pp. 1-7.

[12] "Morphological operations" , Image Processing Toolbox, The Mathworks, http://www.mathworks.com/access/helpdesk/help/toolbox/ images/index.html

[13] R. Fisher, S. Perkins, A. Walker, E. Wolfart. "Connected Component Labeling", http://homepages.inf.ed.ac.uk/rbf/HIPR2/label .htm, 2003. 